\title{
CRB-65 for the assessment of pneumonia severity: who could ask for more?
}

\section{Santiago Ewig, ${ }^{1}$ Tobias Welte ${ }^{2}$}

According to a widespread consensus reflected in all authoritative guidelines, assessment of severity is the key step in the management of patients with community acquired pneumonia (CAP). It is a major criterion in the decision where to treat a patient and it widely determines the amount of diagnostic workup as well as the selection of initial empiric antimicrobial treatment. Overall, it has major implications in terms of outcomes and costs. Two lines of investigation have resulted in two competing tools of severity assessment: the pneumonia severity index (PSI) ${ }^{1}$ and the CURB score and its modifications (CURB-65, CRB-65). ${ }^{2-5}$ In the meantime, it has become evident that the PSI and the CRB-65 score, as the most simple modification of the original CURB score, perform equivalent in terms of prediction of inhospital death. Both predict death in a class 3 pattern, with mortality rates of approximately $1-3 \%$ (PSI class I-III, CRB-65 class 1), $8-10 \%$ (PSI class IV and CRB-class 2) and 20-30\% (PSI class V and CRB-65 class 3). ${ }^{5}$ Both scores also work reasonably well in ambulatory patients. ${ }^{5}$ However, as the CRB-65 score is far easier to remember and to compute, being based exclusively on variables which can be immediately determined without any technical expense other than blood pressure measurement, this score is the one which will gain general acceptance.

It is intriguing that such a simple rule is able to predict the risk of death so accurately. Nevertheless, in this issue of Thorax, Chalmers and colleagues ${ }^{8}$ provide convincing data from a large population showing that the CRB-65 score can be simplified even more by omitting diastolic

\footnotetext{
${ }^{1}$ Thoraxzentrum Ruhrgebiet, Kliniken für Pneumologie und Infektiologie, EVK Herne und Augusta-KrankenAnstalt Bochum, Bochum, Germany; ${ }^{2}$ Medizinische Hochschule Hannover (MHH), Klinik für Pneumologie, Medizinische Hochschule Hannover, Hannover, Germany Correspondence to: Dr Santiago Ewig, Thoraxzentrum Ruhrgebiet, Kliniken für Pneumologie und Infektiologie, EVK Herne und Augusta-Kranken-Anstalt Bochum, Bergstrasse 26, 44791 Bochum, Germany; ewig@ augusta-bochum.de
}

blood pressure $<60 \mathrm{~mm} \mathrm{Hg}$ as a criterion of hypotension (see page 698). Thus CRB-65 can be applied, checking for age $>65$ years, the presence of new onset pneumonia associated mental confusion, hypotension with systolic blood pressure $<90 \mathrm{~mm} \mathrm{Hg}$ and a respiratory rate $>30 / \mathrm{min}$, applying 1 point for each criterion met, with assignment to risk class 1 for those with no points, risk class 2 for those with 1 or 2 points and risk class 3 for those with 3 or 4 points. Patients meeting risk class 1 would be ideal candidates for ambulatory treatment in the absence of any severe decompensated comorbidity, pneumonia related complication (such as large pleural effusion) or social factors requiring hospital care. The additional simplification achieved by Chalmers and colleagues ${ }^{8}$ is important because increasing simplicity of a prediction rule increases its chance of being accepted and applied in everyday practice.

However, no prediction rule is perfect. One important shortcoming of the CRB-65 rule (as well as of the PSI) is the major role of age. Although age is a strong predictor of death from CAP, it is not a good argument in favour of hospital admission in the absence of other severity criteria. As age $>65$ years implies 1 risk point, all patients aged $>65$ years are categorised as risk class 2 at least, and are excluded from the low risk class which suggests ambulatory treatment. Recent data indicate that the majority of acutely ill elderly patients prefer home treatment. Moreover, patients treated in a hospitalat-home model of care had fewer procedures, similar quality of care, shorter treatment stays, lower costs and fewer complications. ${ }^{9}$ Thus although the statistical risk of death is clearly independently increased by age, it should not be the sole criterion for hospital admission.

A very important group with a very high risk of CAP (or nursing home acquired pneumonia) is the very elderly and severely disabled population. In this group, inclusion of functional status may result in superior predictions of death compared with current severity scores. ${ }^{10} 11$
However, a principal concern is whether the prediction of death is an appropriate criterion for hospitalisation, particularly in this population. Several recent reports have shown that adverse outcomes are not restricted to the risk of death but include persistent deteriorations in performance state in these populations. Generic risk factors for functional decline after hospitalisation have been identified, including increasing age, admission mental status as well as overall functional status. ${ }^{12}$ Accordingly, El Solh et al demonstrated that in 301 consecutive patients hospitalised for CAP (mean age 74 (5) years), 36\% of patients developed functional decline at discharge and 11\% had persistent functional impairment at 3 months. Lack of recovery in functional status at 3 months was associated with impaired cognitive ability and preadmission comorbidities. The Charlson comorbidity index was the best predictor of loss of functionality at the time of hospital discharge.$^{13}$ In addition, it could be shown that in nursing home residents, all patients lost function after hospital admission, with loss being even more pronounced in the functionally independent group. ${ }^{14}$

The relevant issue in this context is whether these patients with adverse functional outcomes would have done better if treated under home conditions. Several data support this hypothesis. It has been shown that for residents with low and medium mortality risk, nursing home treatment is likely to be safe and less costly. ${ }^{15}{ }^{16}$ Using a clinical pathway for onsite treatment of pneumonia and other lower respiratory tract infections in nursing homes based on use of oral antimicrobials, portable chest radiographs, oxygen saturation monitoring, rehydration and close monitoring by a research nurse, clinical outcomes were comparable with a usual strategy, including frequent hospitalisation. ${ }^{17}$ Moreover, in one study, treatment for pneumonia without hospital transfer resulted in even better 2 month outcomes compared with hospital treatment. The benefits of long term care facility treatment appeared to be greatest for those with less severe pneumonia and more independent functional status. ${ }^{18}$ Dependent functional status may be a good marker for those unlikely to benefit from hospital transfer. ${ }^{16}$ On the other hand, factors such as oral antimicrobial treatment failure may be important to consider in favour of hospitalisation. ${ }^{19}$ 
Therefore, the challenge is to reconstruct the CRB-65 score into a tool more valuable for this population, providing not only good prediction of the risk of death from CAP but also of the risk of hospitalisation in terms of functional deterioration. The decision to hospitalise will then be made based on this tool and on the background of the quality of onsite facilities for pneumonia treatment ${ }^{917}$ and possible DNR decisions. ${ }^{20}$

Another important issue to consider in the assessment of pneumonia severity is the incorporation of new biomarkers in refining prediction rules. ${ }^{21}$ Several biomarkers are being evaluated in CAP, including procalcitonin (PCT), ${ }^{22}{ }^{23}$ proadrenomedul$\operatorname{lin}^{24}$ and copeptin. ${ }^{25}$ All of these biomarkers have performed well. However, what is the real progress in determining biomarkers? Can they replace clinical rules or even overrule clinical judgement? Most recently, the CAPNETZ investigators demonstrated that the CRB-65 score and PCT were equally predictive of inhospital death of CAP but obviously both predictors did not measure the same. Using $0.228 \mathrm{ng} / \mathrm{ml}$ as the cut-off, deaths were highly more probable in those patients with PCT values above the cut-offs across all CRB-65 risk classes. ${ }^{23}$ If these data were validated and supported in a large population, this would be an ideal strategy to increase the amount of candidates for ambulatory treatment with very low risk of death despite elevated CRB-65 scores. At the same time, it would be a great paradigm of how basic clinical information can be refined by highly sophisticated but rapidly available laboratory investigations.

If we look back, impressive progress has been made in severity assessment of CAP, and CRB-65 seems to be a near ideal tool to help the clinician to validate his clinical judgment. If we look forward, important challenges have to be faced. These include a thorough refinement of CRB-65 by incorporation of functional parameters and reconstruction of CRB-65 for the very elderly and severely disabled population in order not only to assess the risk of death but also to prevent potential harm from hospitalisation. On the other hand, attempts to incorporate biomarkers into clinical prediction rule based algorithms for the site of care seem promising. Who could ask for more?

Competing interests: None.

Thorax 2008;63:665-666. doi:10.1136/thx.2008.099028

\section{REFERENCES}

1. Fine MJ, Auble TE, Yealy DM, et al. A prediction rule to identify low-risk patients with community-acquired pneumonia. N Engl J Med 1997;336:243-50.

2. Lim WS, Lewis S, Macfarlane JT. Severity prediction rules in community acquired pneumonia: a validation study. Thorax 2000;55:219-23.

3. Lim WS, van der Eerden MM, Laing $R$, et al. Defining community acquired pneumonia severity on presentation to hospital: an international derivation and validation study. Thorax 2003;58:377-82.

4. Ewig S, de Roux A, Bauer T, et al. Validation of predictive rules and indices of severity for community acquired pneumonia. Thorax 2004;59:421-7.

5. Bauer TT, Ewig S, Marre R, et al. The CAPNETZ Study Group: CRB-65 predicts death from communityacquired pneumonia. J Intern Med 2006;260:93-101.

6. Capelastegui A, España PP, Quintana JM, et al. Validation of a predictive rule for the management of community-acquired pneumonia. Eur Respir $J$ 2006:27:151-7.

7. Ewig S, Torres A, Woodhead M. Assessment of pneumonia severity: a European perspective. Eur Respir J 2006;27:6-8.

8. Chalmers JD, Singanayagam A, Hill AT. Systolic blood pressure is superior to other haemodynamic predictors of outcome in community acquired pneumonia. Thorax 2008;63:698-702.

9. Leff B, Burton L, Mader SL, et al. Hospital at home: feasibility and outcomes of a program to provide hospital-level care at home for acutely ill older patients. Ann Intern Med 2005;143:798-808.

10. Torres $\mathbf{O H}$, Muñoz J, Ruiz D, et al. Outcome predictors of pneumonia in elderly patients: importance of functional assessment. J Am Geriatr Soc 2004;52:1603-9.

11. Inouye SK, Peduzzi PN, Robison JT, et al. Importance of functional measures in predicting mortality among older hospitalized patients. JAMA 1998;279:1187-93.
12. Sager MA, Rudberg MA, Jalaluddin M, et al. Hospital admission risk profile (HARP): identifying older patients at risk for functional decline following acute medical illness and hospitalization. J Am Geriatr Soc 1996;:44:251-7.

13. El Solh A, Pineda $L$, Bouquin $P$, et al. Determinants of short and long term functional recovery after hospitalization for community-acquired pneumonia in the elderly: role of inflammatory markers. BMC Geriatr 2006;6:12.

14. Mody L, Sun R, Bradley SF. Assessment of pneumonia in older adults: effect of functional status. J Am Geriatr Soc 2006;54:1062-7.

15. Kruse RL, Mehr DR, Boles KE, et al. Does hospitalization impact survival after lower respiratory infection in nursing home residents? Med Care 2004; 42:860-70.

16. Fried TR, Gillick MR, Lipsitz LA. Whether to transfer? Factors associated with hospitalization and outcome of elderly long-term care patients with pneumonia. J Gen Intern Med 1995;10:246-50.

17. Loeb M, Carusone SC, Goeree R, et al. Effect of a clinical pathway to reduce hospitalizations in nursing home residents with pneumonia: a randomized controlled trial. JAMA 2006:295:2503-10.

18. Fried TR, Gillick MR, Lipsitz LA. Short-term functional outcomes of long-term care residents with pneumonia treated with and without hospital transfer. J Am Geriatr Soc 1997;45:302-6.

19. Degelau J, Guay D, Straub K, et al. Effectiveness of oral antibiotic treatment in nursing home-acquired pneumonia. J Am Geriatr Soc 1995:43:245-51

20. Marrie TJ, Fine MJ, Kapoor WN, et al. Communityacquired pneumonia and do not resuscitate orders. J Am Geriatr Soc 2002:50:290-9.

21. Christ-Crain $\mathbf{M}$, Müller B. Biomarkers in respiratory tract infections: diagnostic guides to antibiotic prescription, prognostic markers and mediators. Eur Respir J 2007;30:556-73.

22. Christ-Crain $\mathbf{M}$, Stolz D, Bingisser R, et al Procalcitonin guidance of antibiotic therapy in community-acquired pneumonia: a randomized trial. Am J Respir Crit Care Med 2006;174:84-93.

23. Krüger S, Ewig S, Marre R, et al on behalf of the CAPNETZ study group. Procalcitonin predicts patients at low risk of death from community-acquired pneumonia across all CRB-65 classes. Eur Respir J 2008:31:349-55

24. Christ-Crain M, Morgenthaler NG, Stolz D, et al. Proadrenomedullin to predict severity and outcome in community-acquired pneumonia. Crit Care 2006;10:R96

25. Krüger S, Papassotiriou J, Marre R, et al. CAPNETZ Study Group. Pro-atrial natriuretic peptide and provasopressin to predict severity and prognosis in community-acquired pneumonia: results from the German competence network CAPNETZ. Intensive Care Med 2007;33:2069-78.

\section{The place of varenicline in smoking cessation treatment}

\section{Paul Aveyard}

In this issue of Thorax Aubin and colleagues $^{1}$ report a further trial from the Varenicline Phase III Programme (see

Correspondence to: Dr Paul Aveyard, Division of Primary Care and Public Health, University of Birmingham, Birmingham B15 2T, UK; p.n.aveyard@bham.ac.uk page 717). The trials supporting registration contrasted bupropion with varenicline in a double placebo design. ${ }^{2}{ }^{3}$ This study examines the efficacy of varenicline against nicotine replacement therapy (NRT). In many countries, including the $\mathrm{UK}$, bupropion is rarely used and NRT is the predominant treatment offered in general practices and in specialist smoking cessation clinics. It is not practical to obtain placebo NRT, so this trial was of an open-label design. This publication follows a study by Stapleton et al ${ }^{4}$ with historical controls which showed that varenicline is superior to NRT in achieving abstinence and in reducing withdrawal phenomena such as urges to smoke and withdrawal symptoms.

Varenicline is licensed for smoking cessation around the world, but in the UK the National Institute for Health and Clinical Excellence (NICE) makes 